\title{
Conducción de calor en estado transitorio
}

\section{Transient heat conduction}

\author{
Martín Ortiz-Domínguez ${ }^{a}$, Arturo Cruz-Avilés ${ }^{b}$, Jorge Zuno-Silva $^{c}$, Cesar Mendoza-Gómora ${ }^{d}$, \\ Carlos E. Borja-Soto ${ }^{e}$
}

\begin{abstract}
:
Newton's Law of Cooling or Newtonian Cooling states that the rate of heat loss from a body is proportional to the difference in temperatures between the body and its surroundings. The present work estimates the cooling rate of a warm body in a colder environment, whose temperature is proportional to the difference between the instantaneous body temperature and that of the environment.
\end{abstract}

\section{Keywords:}

Heat transfer, convection coefficient, temperature

\section{Resumen:}

La ley de enfriamiento de Newton o enfriamiento newtoniano establece que la tasa de pérdida de calor de un cuerpo es proporcional a la diferencia de temperaturas entre el cuerpo y sus alrededores. En el presente trabajo se proporciona una estimación de la velocidad de enfriamiento de un cuerpo cálido en un ambiente más frío, cuya temperatura es proporcional a la diferencia entre la temperatura instantánea del cuerpo y la del ambiente.

\section{Palabras Clave:}

Transferencia de calor, coeficiente de convección, temperatura

\section{Introducción}

La termodinámica trata de la cantidad de transferencia de calor a medida que un sistema pasa por un proceso de un estado de equilibrio a otro y no hace referencia a cuánto durará ese proceso. Pero en la ingeniería a menudo estamos interesados en la rapidez o razón de esa transferencia, la cual constituye el tema de la ciencia de la transferencia de calor. La transferencia de calor es el paso de energía térmica desde un cuerpo de mayor

\footnotetext{
a Autor de Correspondencia, Licenciatura en Ingeniería Mecánica, Escuela Superior de Ciudad Sahagún, UAEH, Tepeapulco, Hidalgo, México, ORCID: 0000-0003-4475-9804, Email: martin_ortiz@uaeh.edu.mx;

b Licenciatura en Ingeniería Mecánica, Escuela Superior de Ciudad Sahagún, UAEH, Tepeapulco, Hidalgo, México, ORCID: 0000-00030455-1646, Email: arturo_cruz8085@uaeh.edu.mx;
}

c Licenciatura en Ingeniería Mecánica, Escuela Superior de Ciudad Sahagún, UAEH, Tepeapulco, Hidalgo, México, https://orcid.org/00000002-1997-5399, Email: jorge_zuno@uaeh.edu.mx;

d Licenciatura en Ingeniería Mecánica, Escuela Superior de Ciudad Sahagún, UAEH, Tepeapulco, Hidalgo, México, https://orcid.org/0000-0003-1462-0588.Email: cesar_mendoza@uaeh.edu.mx;

e Licenciatura en Ingeniería Mecánica, Escuela Superior de Ciudad Sahagún, UAEH, Tepeapulco, Hidalgo, México, https://orcid.org/00000003-3385-8348. Email: carlos_borja@uaeh.edu.mx; 
temperatura a otro de menor temperatura (ver Figura 1). Cuando un cuerpo, por ejemplo, un objeto sólido o un fluido, está a una temperatura diferente de la de su entorno u otro cuerpo, la transferencia de energía térmica, también conocida como transferencia de calor o intercambio de calor, ocurre de tal manera que el cuerpo y su entorno alcancen equilibrio térmico.

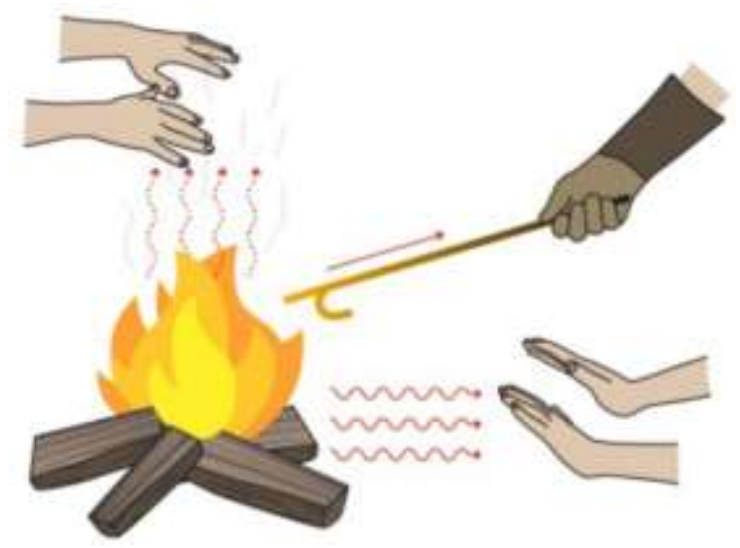

Figura 1. Transferencia de calor. Fuente: (Carlos,2013)

Con base en la experiencia, se sabe que una bebida enlatada fría dejada en una habitación se entibia y una bebida enlatada tibia que se deja en un refrigerador se enfría. Esto se lleva a cabo por la transferencia de energía del medio caliente hacia el frío. La transferencia de energía siempre se produce del medio que tiene la temperatura más elevada hacia el de temperatura más baja y esa transferencia se detiene cuando ambos alcanzan la misma temperatura. El requisito básico para la transferencia de calor es la presencia de una diferencia de temperatura. No puede haber transferencia neta de calor entre dos medios que están a la misma temperatura. La diferencia de temperatura es la fuerza impulsora para la transferencia de calor, precisamente como la diferencia de tensión es la fuerza impulsora para el flujo de corriente eléctrica y la diferencia de presión es la fuerza impulsora para el flujo de fluidos. La velocidad de la transferencia de calor en cierta dirección depende de la magnitud del gradiente de temperatura (la diferencia de temperatura por unidad de longitud o la razón de cambio de la temperatura en esa dirección). A mayor gradiente de temperatura, mayor es la razón de la transferencia de calor. Existen tres mecanismos básicos de la transferencia de calor: la conducción, la convección y la radiación, y se discute la conductividad térmica. La convección es el modo de transferencia de calor entre una superficie sólida y el líquido o gas adyacentes que están en movimiento, y comprende los efectos combinados de la conducción y del movimiento del fluido. 1-10

\section{Objetivo general}

Determinar la temperatura de un fluido caliente después de un periodo de tiempo a través de la ley de enfriamiento de Newton para aplicaciones industriales.

\section{Objetivos específicos}

- Deducir la temperatura superficial $(T)$ del objeto o fluido en función del tiempo, a través del balance de energía, para su comprobación experimental.

- Simular la temperatura superficial $(T)$ del objeto o fluido en función del tiempo, a través del software SOLIDWORKS $^{\circledR}$ Flow Simulation, para su comprobación experimental.

\section{Aplicaciones prácticas}

- En la actualidad el enfriamiento newtoniano es utilizado especialmente en modelos climáticos como una forma rápida y menos cara computacionalmente de calcular la evolución de temperatura de la atmósfera (ver Figura 2). Estos cálculos son muy útiles para determinar las temperaturas, así como para predecir los acontecimientos de los fenómenos naturales.

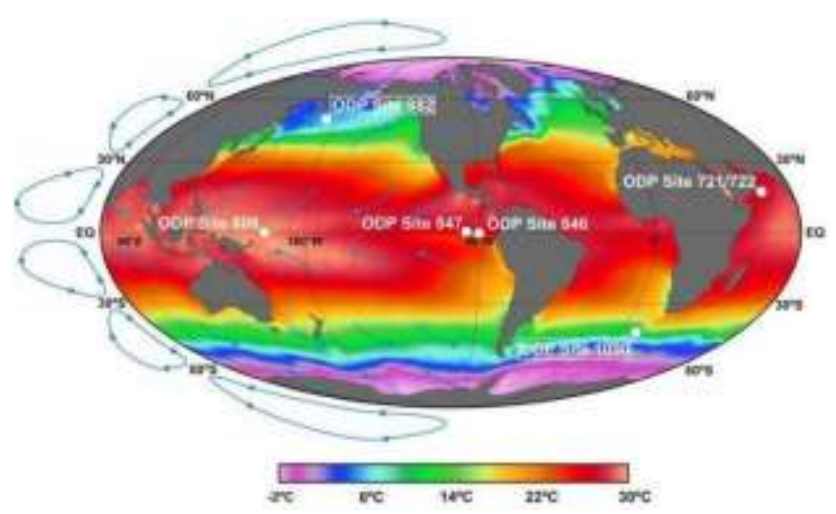

Figura 2. Temperatura de la atmósfera. Fuente: (Martínez García et al. 2010) 
- En el procesador (ver Figura 3), el control de la temperatura es fundamental, conociendo la temperatura del ambiente y el tiempo que toma alcanzar una temperatura moderada se puede saber cuánto tiempo de vida tiene un procesador (teóricamente).

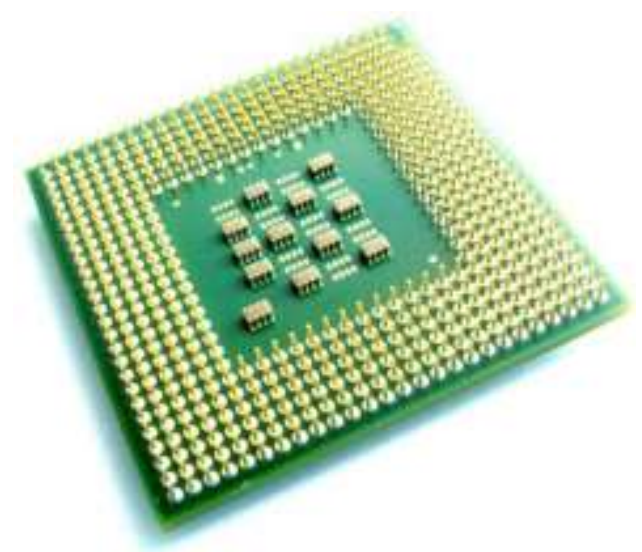

Figura 3. Procesador de computadora. Fuente: (Schmidt, 2014)

Los sistemas de enfriamiento (ver Figura 4) ya sean por aire o agua permiten la extracción de calor de los componentes, lógicamente, mientras menos tiempo les tome extraer el calor, mejor serán estos.

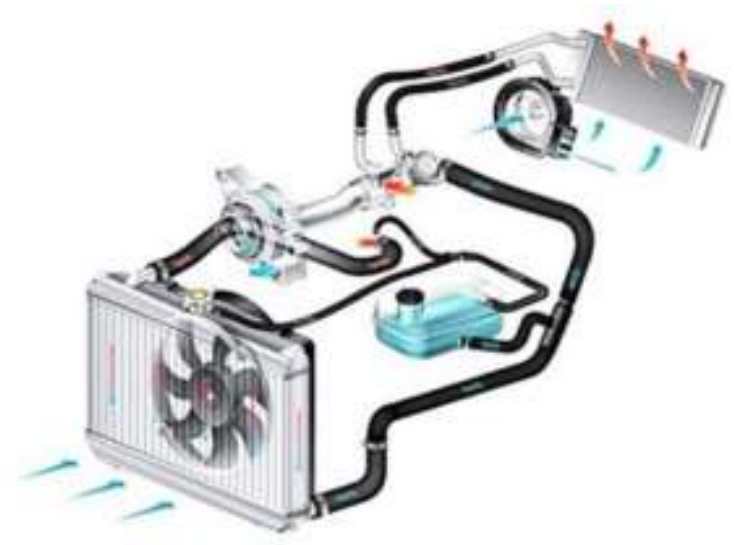

Figura 4. Sistema de enfriamiento automotriz. Fuente: (Federico, 2018)

\section{Teoría}

Si la temperatura del cuerpo es mayor que la ambiental, entonces deberá experimentar una pérdida de calor, la cual será proporcional a la diferencia de temperaturas, podemos expresar esto en forma de una ecuación diferencial como:

$$
d Q=-C_{p} d T
$$

La Ec. (1), también se puede expresar como:

$$
d Q=-m c_{P} d T
$$

Donde $Q$ es el calor, $T$ es la temperatura, $m$ es la masa del cuerpo y su calor específico $c_{P}$, el signo menos indica una pérdida calorífica. Por otro lado, los procesos de transferencia de calor que comprenden cambio de fase de un fluido también se consideran como convección a causa del movimiento de ese fluido inducido durante el proceso, como la elevación de las burbujas de vapor durante la ebullición o la caída de las gotitas de líquido durante la condensación. A pesar de la complejidad de la convección, se observa que la rapidez de la transferencia de calor por convección es proporcional a la diferencia de temperatura y se expresa en forma conveniente como:

$$
\dot{Q}=h A_{s}\left(T-T_{\infty}\right)
$$

Donde $h$ es el coeficiente de transferencia de calor por convección, en $\mathrm{W} / \mathrm{m}^{2}{ }^{\circ} \mathrm{C}, A_{s}$ es el área superficial a través de la cual tiene lugar la transferencia de calor por convección, $T$ es la temperatura de la superficie y $T_{\infty}$ es la temperatura del ambiente. El coeficiente de transferencia de calor por convección $h$ no es una propiedad del fluido. Es un parámetro que se determina en forma experimental y cuyo valor depende de todas las variables que influyen sobre la convección, como la configuración geométrica de la superficie, la naturaleza del movimiento del fluido, las propiedades de éste y la velocidad masiva del mismo. Reescribiendo la Ec. (3), tenemos: 


$$
\frac{d Q}{d t}=h A_{s}\left(T-T_{\infty}\right)
$$

Separando variables en la Ec. (4):

$$
d Q=h A_{s}\left(T-T_{\infty}\right) d t
$$

Podemos combinar las Ecs. (2) y (5), de la siguiente manera:

$$
-m c_{P} d T=h A_{s}\left(T-T_{\infty}\right) d t
$$

Aplicando la técnica de variables separables:

$$
\frac{d T}{T-T_{\infty}}=-\frac{h A_{s}}{m c_{P}} d t
$$

Reescribiendo la Ec. (7), tenemos:

$$
\frac{d T}{T-T_{\infty}}=-\kappa d t
$$

Donde $\kappa=h A_{s} / m c_{P} \quad$ (Kappa) es una constante de proporcionalidad conocida como parámetro de enfriamiento. Resolviendo la ecuación diferencial para un cuerpo que se enfría desde una temperatura $T_{c}$ (temperatura caliente) hasta una temperatura $T$, obtenemos la temperatura del objeto o fluido en función del tiempo:

Integrando la Ec. (8), con los límites correspondientes, tenemos:

$$
\ln \left(T-T_{\infty}\right)-\ln \left(T_{c}-T_{\infty}\right)=-\kappa t
$$

Tomando el exponencial en ambos lados de la Ec. (11), resulta en:

$$
\ln \left(\frac{T-T_{\infty}}{T_{c}-T_{\infty}}\right)=-\kappa t
$$

$$
\exp \left(\ln \left(\frac{T-T_{\infty}}{T_{c}-T_{\infty}}\right)\right)=\exp (-\kappa t)
$$

Despejando a la temperatura $T$ de la Ec. (13), obtenemos:

$$
T=T_{\infty}+\left(T_{c}-T_{\infty}\right) \exp (-\kappa t)
$$

\section{Materiales y procedimiento}

En este experimento, determinaremos la temperatura superficial de enfriamiento de un fluido calentado a una temperatura $T_{c}$ previamente seleccionada con respecto al tiempo.

1. Vaso precipitado: sirve para preparar disoluciones, calentar, triturar, guardar líquidos (ver Figura 5).

$$
\int_{T=T_{C}}^{T=T} \frac{d T}{T-T_{\infty}}=-\kappa \int_{t=0}^{t=t} d t
$$




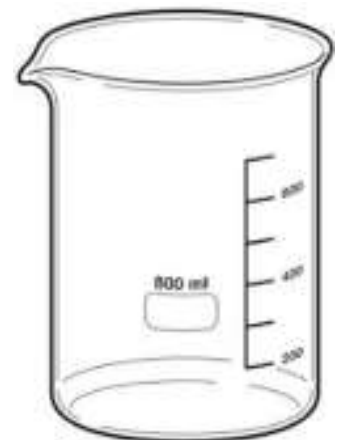

Figura 5. Vaso precipitado. Fuente: (Materiales Laboratorio, s.f.)

2. Termómetro de mercurio (ver Figura 6) es un tipo de termómetro empleado para medir las temperaturas del material seleccionado.

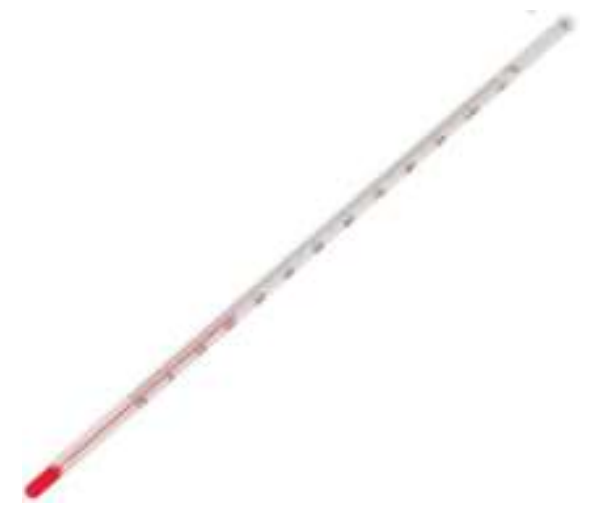

Figura 6. Termómetro de mercurio. Fuente: (Reachem, 2020)

3. Cilindro de gas para contener gas LP (ver Figura 7).

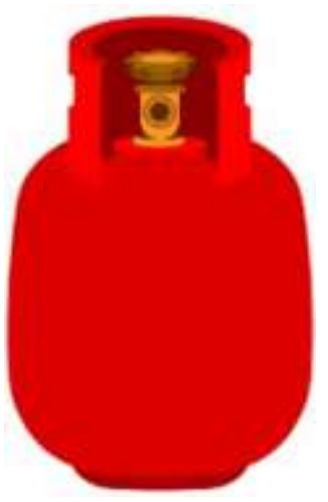

Figura 7. Cilindro de gas. Fuente: (Vexeles, 2017)

4. Mechero: es la principal fuente de calor en un laboratorio (ver Figura 8).

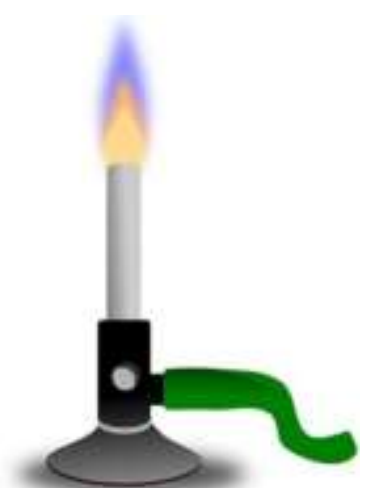

Figura 8. Mechero. Fuente: (Materiales de laboratorio, 2020)

5. Trípode: es una pieza metálica para colocar la rejilla metálica y realizar calentamiento (ver Figura 9). 


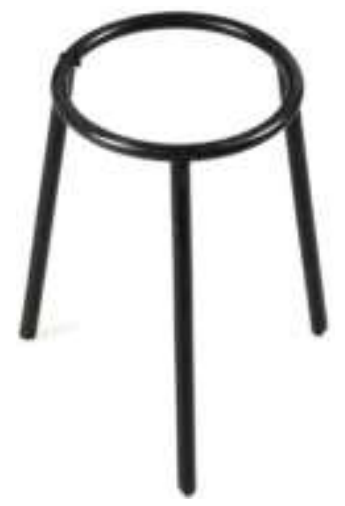

Figura 9. Trípode. Fuente: (Materiales de laboratorio, 2020)

6. Pinza para soporte: se usa para sujetar instrumentos en el montaje de sistemas (ver Figura 10).

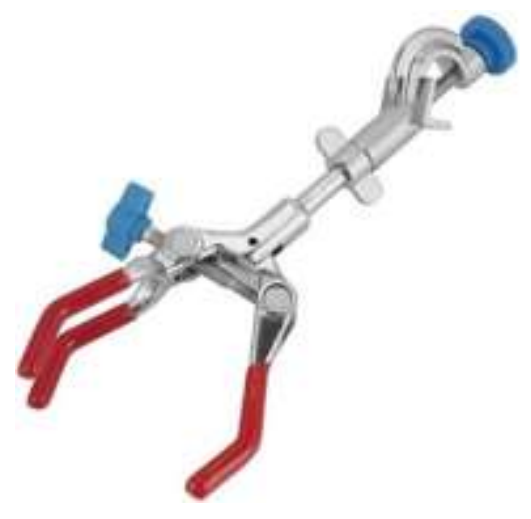

Figura 10. Pinza. Fuente: (Materiales de laboratorio, 2020)

7. Rejilla metálica con centro de amianto: se utiliza para calentar indirectamente ya que la llama del mechero se concentra en el amianto (ver Figura 11).

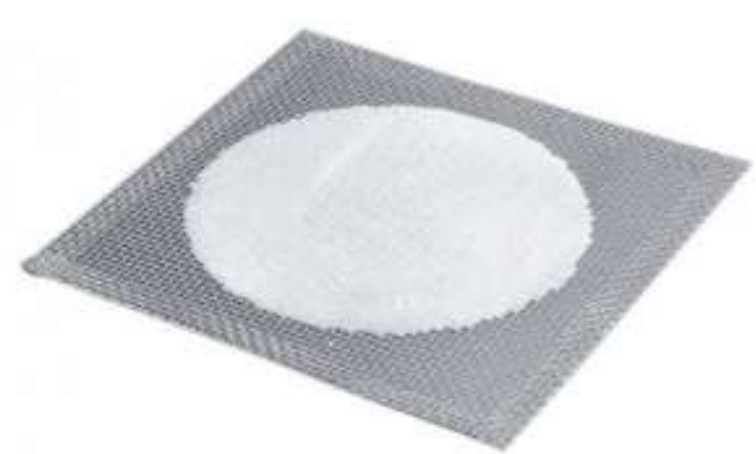

Figura 11. Rejilla metálica con centro de amianto. Fuente: (Materiales de laboratorio, 2020)

8. Soporte universal: es una pieza básica en el montaje de los sistemas y aparatos, para fijar pinzas y anillos de hierro (ver Figura 12).

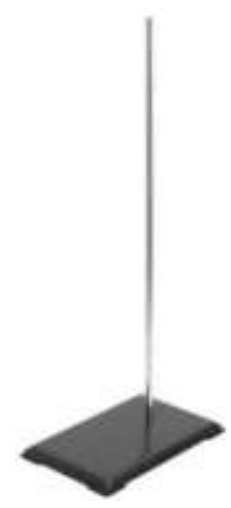

Figura 12. Soporte universal. Fuente: (Materiales de laboratorio, 2020)

9. Cronómetro: es un reloj de mano diseñado para medir la cantidad de tiempo que transcurre entre su activación y desactivación (ver Figura 13). 


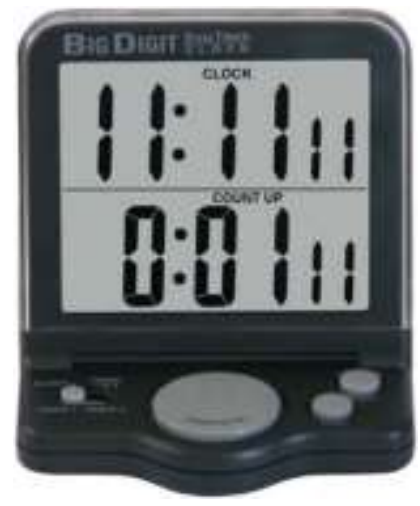

Figura 13. Cronómetro digital. Fuente: (The motorsport, 2020)

En el esquema se observa el dispositivo empleado con todos los componentes para comprobar la ley de enfriamiento de Newton (ver Figura 14).

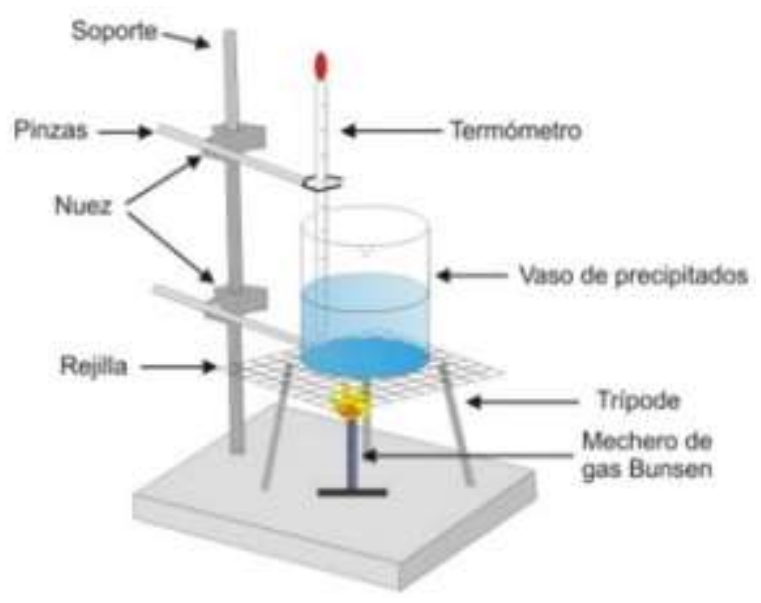

Figura 14. Esquema del dispositivo. Fuente: Elaboración propia.

Equipo de protección individual a utilizar:

- Bata blanca

- Botas de seguridad

- Guantes de protección térmica

- Gafas de protección
Realice el experimento siguiendo los siguientes pasos:

- Limpie el vaso de precipitado y agregue $200 \mathrm{ml}$ de agua limpia a temperatura ambiente $\left(T_{\infty}\right)$.

- Introduzca el termómetro con ayuda de las pinzas para soporte hasta que el bulbo apenas quede cubierto por el agua.

- Registre la temperatura que marca el termómetro.

- Abrir la llave de paso del gas.

- Encender el mechero con una cerilla. Si tiene dispositivo de seguridad ("corta-gas"), mantener presionado el pulsador de la válvula hasta que la llama sea fija.

- Cuando el termómetro registre aproximadamente la temperatura de $T_{c}=90^{\circ} \mathrm{C}$, apagar el mechero, cerrar su llave negra y la del gas individual.

- Inmediatamente empiece a registrar el tiempo con ayuda de un cronómetro y deténgalo hasta que el termómetro registre una temperatura de $T=85^{\circ} \mathrm{C}$.

- Repita el paso anterior y detenga el cronómetro y deténgalo hasta que el termómetro registre una temperatura de $T=80^{\circ} \mathrm{C}$.

- Repita el paso anterior y detenga el cronómetro y deténgalo hasta que el termómetro registre una temperatura de $T=75^{\circ} \mathrm{C}$.

- Repita el paso anterior y detenga el cronómetro y deténgalo hasta que el termómetro registre una temperatura de $T=70^{\circ} \mathrm{C}$.

\section{Resultados}

Tabla 1. Temperaturas experimentales en diferentes tiempos.

Fuente: Elaboración propia

\begin{tabular}{|c|c|}
\hline $\begin{array}{c}\text { Temperatura } \\
\text { experimental } \\
\left({ }^{\circ} \mathrm{C}\right)\end{array}$ & $\begin{array}{c}\text { Tiempo } \\
\text { (min) }\end{array}$ \\
\hline $85^{\circ} \mathrm{C}$ & \\
\hline $80^{\circ} \mathrm{C}$ & \\
\hline $75^{\circ} \mathrm{C}$ & \\
\hline $70^{\circ} \mathrm{C}$ & \\
\hline
\end{tabular}


Temperatura de $75 \stackrel{\circ}{\circ} \mathrm{C}$ alcanzados en la simulación

\section{Cálculos}

Registre las siguientes dimensiones:

Densidad del agua

$\rho=997 \mathrm{~kg} / \mathrm{m}^{3}$

Volumen del agua

$V=200 \mathrm{ml}=0.0002 \mathrm{~m}^{3}$

Área superficial del recipiente

$A_{s}=0.00363 m^{2}$

Calor específico

$c_{P}=4186 \mathrm{~J} / \mathrm{kg} \cdot{ }^{\circ} \mathrm{C}$

Temperatura caliente (inicial)

$T_{c}=90^{\circ} \mathrm{C}$

Temperatura ambiente

$T_{\infty}=22^{\circ} \mathrm{C}$

Temperaturas que queremos alcanzar

$T=85^{\circ} \mathrm{C}, 80^{\circ} \mathrm{C}, 75^{\circ} \mathrm{C}, 70^{\circ} \mathrm{C}$

Coeficiente de convección

$h=169.31762 \mathrm{~W} / \mathrm{m}^{2} \cdot{ }^{\circ} \mathrm{C}$

Masa

$m=\rho V$

Con ayuda del software SOLIDWORKS® Flow Simulation es una intuitiva solución de dinámica de fluidos computacional (CFD, del inglés "Computational Fluid Dynamics") integrada en SOLIDWORKS 3D CAD que le permite simular de forma rápida y sencilla flujos de líquido y gas a través y alrededor de sus diseños para calcular así el rendimiento y las capacidades del producto. Se determina la temperatura de enfriamiento con respecto a un tiempo determinado.

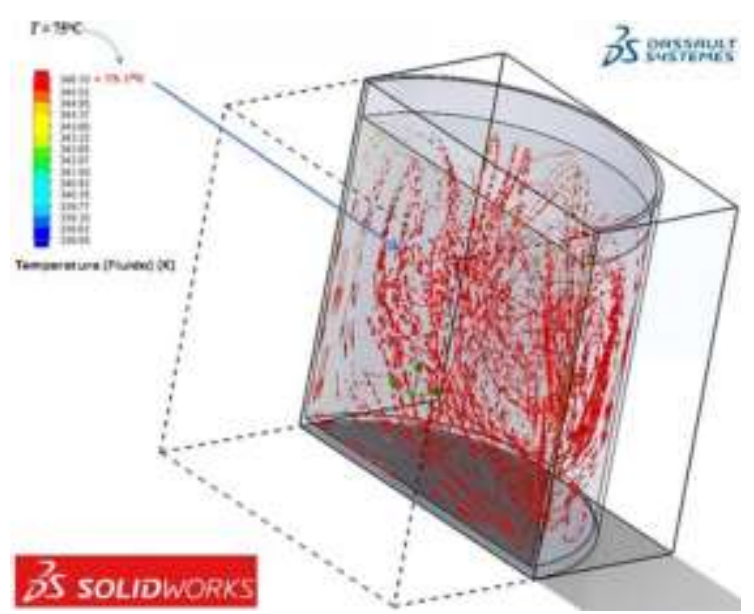

Figura 15. Simulación en SolidWorks. Fuente: Elaboración propia

Asimismo, con ayuda de la Ec. (14), complete la Tabla 2.

Tabla 2. Comparación de temperaturas experimentales y teóricas obtenidas en diferentes tiempos.

Fuente: Elaboración propia

\begin{tabular}{|c|c|c|c|}
\hline $\begin{array}{c}\text { Temperatura } \\
\text { experimental } \\
\left({ }^{\circ} \mathrm{C}\right)\end{array}$ & $\begin{array}{c}\text { Temperatura } \\
\text { teórica } \\
\left(\begin{array}{c}\text { Ec. }(14)) \\
\left({ }^{\circ} \mathrm{C}\right)\end{array}\right.\end{array}$ & $\begin{array}{c}\text { Temperatura } \\
\text { simulada } \\
\text { con } \\
\text { SolidWorks } \\
\left({ }^{\circ} \mathrm{C}\right)\end{array}$ & $\begin{array}{c}\text { Tiempo } \\
(\mathrm{min})\end{array}$ \\
\hline $85^{\circ} \mathrm{C}$ & & & \\
\hline $80^{\circ} \mathrm{C}$ & & & \\
\hline $75^{\circ} \mathrm{C}$ & & & \\
\hline $70^{\circ} \mathrm{C}$ & & & \\
\hline
\end{tabular}

\section{Conclusiones}

Determinamos una expresión matemática para estimar la temperatura de un fluido caliente después de un periodo de tiempo a través de la ley de enfriamiento de Newton.

\section{Agradecimientos}

El trabajo descrito en el presente artículo fue financiado por la beca otorgada por PROMEP. Asimismo, le agradezco al Dr. Jorge Zuno Silva, quien es Director de 
la Escuela Superior de Ciudad Sahagún-UAEH, México, por todas las facilidades para concluir este trabajo.

\section{Referencias}

[1] Çengel, Y. A., \& Cimbala, J. M. (2018). Mecánica de fluidos: Fundamentos y aplicaciones / Yunus A. Çengel y John M. Cimbala México D.F.: McGraw Hill

[2] White, F., (2018), Mecánica de fluidos, Ed. McGraw Hill

[3] Barrero Ripoll, A., Pérez-Saborid Sánchez-Pastor, M., (2005) Fundamentos y aplicaciones de la Mecánica de Fluidos, Ed. McGraw Hill

[4] Bauman R. P, (2000), An alternative derivation of Bernoulli's principle. Am. J. Phys. 68 (3), p. 288-289

[5] Walker J., (1998), The hydrostatic paradox: simple geometries, explicit calculations. The Physics Teacher, Vol. 36, pp. 378-379.

[6] Wilson A., (1995), The hydrostatic paradox. The Physics Teacher, Vol. 33, pp. 538-539.

[7] Gaffney C., (2000), The hydrostatics of trapped bubbles in fluids. The Physics Teacher, vol 38, pp. 458-460.

[8] Franco A., (1982), Analogías Físicas (Mecánica, Electricidad, Fluidos). Documentación E.I. (Enseñanzas Integradas). Vol 6 (3), págs. 63-69

[9] Doltz M, Hernández M J, Delegido J, Casanovas A., (2006), A laboratory experiment on inferring Poiseuille's law for undergraduate students. Eur. J. Phys. 27; 1083-1089

[10] Mott, R.: «Mecánica de fluidos aplicada». Ed. Prentice Hall

[11] Carlos, (2013). Radicación, conducción y convección: tres formas de transferencia de calor. Recuperado de: https://nergiza.com/radiacionconduccion-y-conveccion-tres-formas-de-transferencia-de-calor/

[12] Martínez-García, A., Rosell-Melé, A., McClymont, E. L. Gersonde, R. and Haug, G. H., (2010). Subpolar link to the Emergence of the Modern Equatorial Pacific Cold Tongue, Science: 328.

[13] Schmidt, D. C., (2014). Keeping an Unfair Advantage in a Globalized \& Commoditized Word via Open Systems Architecture, Open Systems Architecture: Progress \& Challenges.

[14] Federico, (2018). Limpieza del Sistema de enfriamiento del vehículo, Recuperado de: https://autoytecnica.com/limpieza-del-sistema-deenfriamiento-del-vehiculo/

[15] Materiales Laboratorio, (s.f.). Vaso de precipitado, Recuperado de: https://materialesdelaboratorio.info/vaso-de-precipitado

[16] Reachem, (2020). Termómetros de mercurio $0^{\circ} \mathrm{C}$ a $360^{\circ} \mathrm{C}$, Recuperado de: https://www.reachem.cl/producto/termometros-mercurio-0c-a360c-clase-b/

[17] Vexeles, (2017). Icono de tanque de gas rojo, recuperado de: https://es.vexels.com/png-svg/vista-previa/149988/icono-de-tanque-degas-rojo

[18] Materiales de laboratorio, (2020). Material para laboratorio de química, recuperado de: https://www.amazon.com.mx/TOPINCNAccesorio-Laboratorio-Giratorio-Condensador/dp/B07NRHSTG4

[19] The motorsport center, (2020). Cronometro digital Big Digital, recuperado de: llystore.net/es/cronometros/1887-cronometro-digitalbig-digit-4891727089500.html 